\title{
Treatment of pre- and confirmed cervical cancer in HIV-seropositive women from developing countries: a systematic review
}

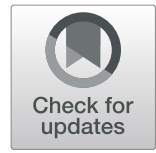

Witness Mapanga ${ }^{1,2,3^{*}}$, Elvira Singh ${ }^{4,5}$, Shingairai A. Feresu ${ }^{6}$ and Brendan Girdler-Brown ${ }^{1}$

\begin{abstract}
Background: Cervical cancer has become a major public health challenge in developing countries with a reported age-standardised incidence rate of about 17.9/100,000/year and lifetime risks approaching 1 in 20 in some settings. Evidence indicates that HIV-seropositive women are 2 to 12 times more likely to develop precancerous lesions that lead to cervical cancer than HIV-negative women. There is a lack of rigorous evidence on which treatment methods are being utilised for HIV-positive women, and this review aims to synthesise available evidence on treatment modalities for both cervical neoplasia and cervical cancer in HIV-seropositive women in developing countries.

Methods: A systematic review guided by a published protocol was conducted. Online databases including MEDLINE/PubMed, Embase, CINAHL and Emerald (via EBSCOhost), PsycINFO, Cochrane Library, and health databases, which cover developing countries (3ie Systematic Reviews, WHO library and databases, World Bank website), were searched for published articles. Additional articles were found through citation, reference list tracking, and grey literature. Study design, treatment category, geographic country/region, and key outcomes for each included article were documented and summarised.
\end{abstract}

Results: Thirteen research articles from sub-Saharan Africa, Asia, and South America were included. Eight (61.5\%) articles focused on the treatment of cervical cancer with the remaining five (38.5\%) assessed cervical neoplasia treatment. The available cervical cancer treatments, radiotherapy, chemotherapy, chemoradiation, and surgery are effective for HIV-seropositive patients, and these are the same treatments for HIV-negative patients. Both cryotherapy and LEEP are effective in reducing CIN2+ among HIV-seropositive women, and a choice between the treatments might be based on available resources and expertise. Radiation, chemotherapy, concurrent treatment using radiotherapy and chemotherapy, and surgery have shown the possibility of effectiveness among HIVseropositive women. Cervical cancer stage, immunosuppressive level including those on HAART, and multisystem toxicities due to treatment are associated with treatment completion, prognostic, and survival outcomes.

(Continued on next page)

\footnotetext{
* Correspondence: witnessmapanga@yahoo.co.uk

${ }^{1}$ School of Health Systems and Public Health, Epidemiology \& Biostatistics, University of Pretoria, 5-10 H.W. Snyman Building, Pretoria, South Africa ${ }^{2}$ Non-Communicable Diseases Research (NCDR) Division of the Wits Health Consortium, Faculty of Health Sciences, University of the Witwatersrand, Johannesburg, South Africa

Full list of author information is available at the end of the article
}

(c) The Author(s). 2020 Open Access This article is licensed under a Creative Commons Attribution 4.0 International License, which permits use, sharing, adaptation, distribution and reproduction in any medium or format, as long as you give appropriate credit to the original author(s) and the source, provide a link to the Creative Commons licence, and indicate if changes were made. The images or other third party material in this article are included in the article's Creative Commons licence, unless indicated otherwise in a credit line to the material. If material is not included in the article's Creative Commons licence and your intended use is not permitted by statutory regulation or exceeds the permitted use, you will need to obtain permission directly from the copyright holder. To view a copy of this licence, visit http://creativecommons.org/licenses/by/4.0/ The Creative Commons Public Domain Dedication waiver (http://creativecommons.org/publicdomain/zero/1.0/) applies to the data made available in this article, unless otherwise stated in a credit line to the data. 


\begin{abstract}
(Continued from previous page)
Conclusions: Treatment of cervical cancer is based on the stage of cancer, and poor outcomes in most developing countries might be due to a lack of optimal treatment regimen. Those infected with HIV were younger and had advanced cervical cancer as compared to those who were HIV-negative. Facilitation and putting HIV-infected people on life-long ART is of importance and has been found to have a positive impact on cervical cancer treatment response. Research on precancerous lesions and cervical cancer management of HIV-seropositive patients focusing on the quality of life of those treated; the effectiveness of the treatment method considering CD4+ count and ART is required.
\end{abstract}

Systematic review registration: PROSPERO CRD42018095707

Keywords: Cervical neoplasia, Cervical cancer, Treatment, HIV, Developing countries

\section{Background}

Persistent infection with human papillomavirus (HPV) is the main risk factor for developing cervical cancer [1-4]. The HIV burden has seen an increase in morbidity and mortality due to cervical cancer, especially in developing countries, where HIV incidence and prevalence are high [5-7]. In these developing countries, the reported agestandardised incidence rate can be as high as 17.9/100, 000/year [8]. Also, most of these patients are mostly diagnosed at an advanced stage due to a lack of coordinated and systematic screening $[9,10]$. The management of both cervical neoplasia and cervical cancer in HIV-seropositive women, who mostly reside in developing countries, is bound to be challenging especially with the potential risk of medical complications, and resource constraints including unavailability of treatments [7]. There are several treatment modalities for cervical cancer, namely, chemotherapy, surgical management, and radiation therapy [7, 11, 12]. Treatment modalities for precancerous lesions and cervical cancer are based on the stage of the lesions and available resources; the associated poor outcomes of treatment among HIV seropositive women in developing countries may be due to lack of optimal treatment regimen $[10,13]$. Most developing countries lack skilled surgeons to carry out radical surgery for cervical cancer, and this has left HIV-seropositive cervical cancer patients with few treatment options. In cases where surgeons are available, surgery is expensive and out of reach of many, who happen to be poor [14]. In developing countries especially sub-Sahara Africa, many women with cervical cancer have no access to radiotherapy, further limiting their treatment options. However, little or no information exists that has shown that any of the current treatments are effective compared to other treatments when it comes to treating cervical cancer in HIV-seropositive women. In subSaharan Africa, treatments like radiation therapy and other surgical procedures are not fully utilised because of lack of equipment and qualified personnel; hence, little has been documented on which treatment procedures are being used for the treatment of cervical cancer in HIV seropositive women [15]. Therefore, there is a lack of evidence-based guidelines and strategies for the treatment of cervical cancer in HIV-seropositive women in most developing countries. Coupled with this, there is little rigorous evidence on the global epidemiology of the treatment of cervical cancer in HIV-seropositive women [15]. This review aims to answer the following questions: What are the treatment modalities that are being used to treat and manage cervical cancer in HIV-seropositive women in developing countries? Are these the same treatment modalities that are being used for HIV-negative women? Are the treatment modalities effective in HIV-seropositive women? This review will investigate and identify the existing treatment modalities used for cervical cancer in HIVseropositive women in some of the developing countries.

\section{Methods}

\section{Search strategy}

This review was guided by a protocol [16] and registered with PROSPERO (CRD42018095707). The PRISMA guidelines (Fig. 1) informed the reporting of this systematic [17]. Two independently working reviewers (WM and SF) searched MEDLINE/PubMed (1966 to present, using Medical Subject Heading (MeSH) terms), Embase (1980 to present) via the OVID interface, and Cochrane, PsycINFO, Emerald, and CINAHL (all from 1961 to present) via EBSCOhost, using a combination of the following words: "Cervical Neoplasm", "Cervical Cancer", "treatment", and "HIV". The initial results (2753 records) were further narrowed down by adding "developing countries" (individual database search strategies are included in Additional file 1). In addition, the two reviewers (WM and SF) also searched the 3ie Systematic Reviews, WHO library and databases, World Bank website and WHO ICTRP and cliniccaltrials.gov [16] and identified an additional 4 studies. All the searches included articles published up to 31 January 2020, which was also the last date of the searches.

\section{Eligibility criteria}

Studies were eligible for inclusion if they investigated cervical neoplasia or cervical cancer treatment methods 


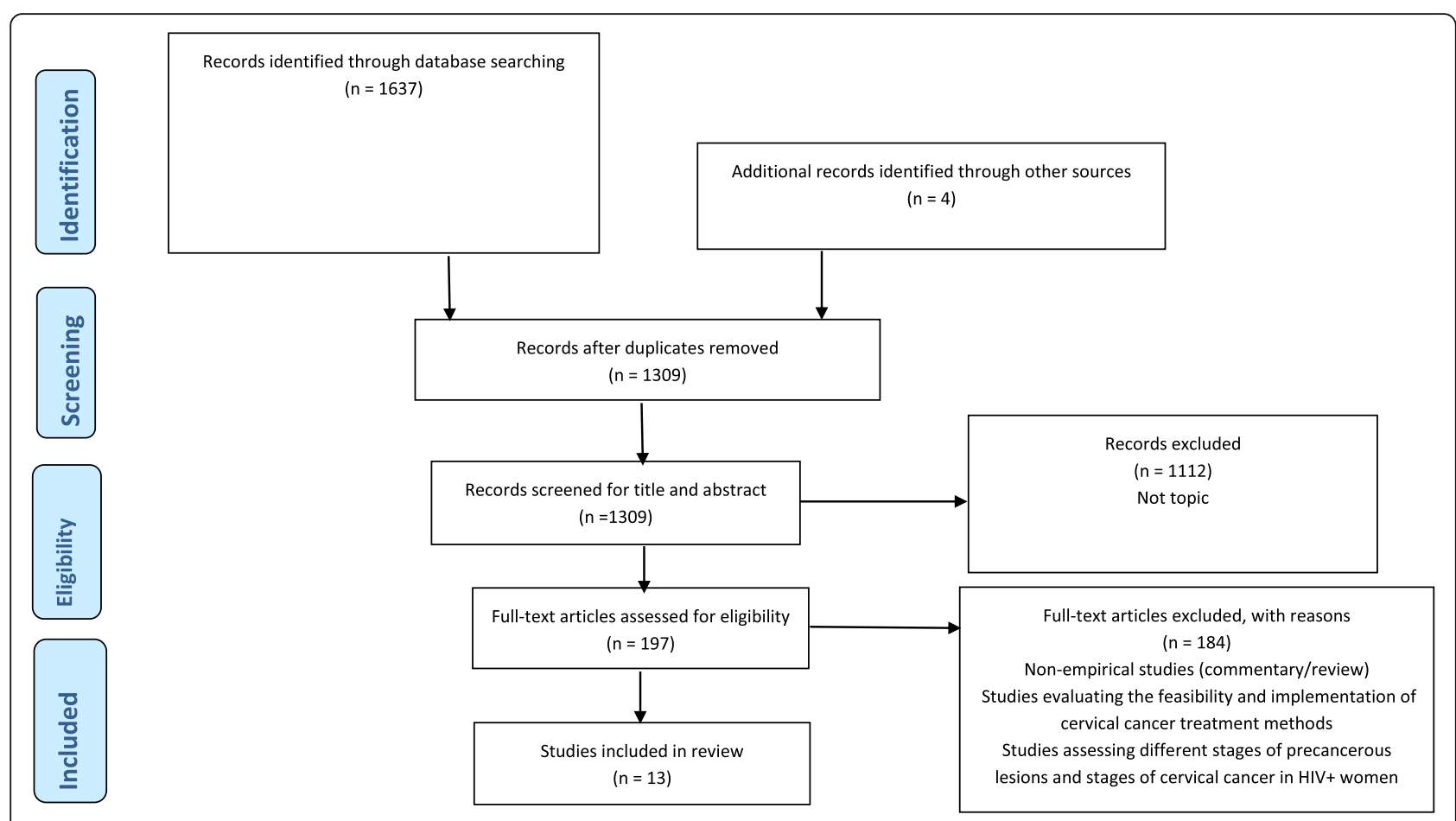

Fig. 1 PRIMSA flowchart. The search strategy is reported according to PRISMA guidelines

(cryotherapy, loop electrosurgical excision procedure (LEEP), chemotherapy, surgery, and radiation therapy among others) for HIV-positive women in developing countries, peer-reviewed, grey literature (dissertations, conference papers, government reports), and done for or in countries or regions considered developing by the United Nations [16]. Studies were excluded if they had unrepresentative samples.

\section{Study selection}

The search of databases and grey literature yielded 1637 results, and an additional four studies were identified through reference tracking to make a total of 1641 articles (Fig. 1). All the articles were combined into EndNote reference management software, and 332 duplicates were removed. The remaining 1309 articles were exported to Covidence software, where duplicate screening was performed. Two independently working reviewers (WM and SF) conducted title and abstract screening based on the relevance to the review question. Studies were excluded when the title and abstract mentioned cervical cancer screening or vaccination or described the implementation process of cervical cancer treatment. Disagreements related to the screening process were resolved as a team through discussions as reported in the published protocol [16]. Through title and abstract screening, 1112 articles were excluded. Two independent reviewers (WM and BGB) conducted fulltext screening on the remaining 197 articles, and 184 articles did not meet the eligibility criteria and were excluded. A total of 13 articles met the eligibility criteria and included in the final analysis (Fig. 1).

\section{Data extraction}

Two independent reviewers (WM and SF) conducted double data extraction in Covidence software on the 13 articles that were included in the final analysis, whilst the rest of the team checked for quality and consistency. A data extraction form, as indicated in the published protocol [16], guided the data extraction process. The quality of the data extraction form was determined by piloting 5 articles and the team discussing and resolving all the inconsistencies through consensus. The following variables were extracted from the studies: first author and publication year, the title of the study, study type, aim of the study, participants and their age, study setting, stage of cervical cancer, treatment method, outcomes, results, and authors' conclusions.

\section{Quality assessment of included studies}

For quality assessment of the studies, the team utilised a combination of the NIH Study Quality Assessment Tools for observational cohort and cross-sectional, case-control, and before-after studies, together with a modified version of the Newcastle-Ottawa Quality Assessment Scale [18, 19]. For the quality assessment process to be easy to conduct, studies were grouped into observational studies without a control group(s), observational studies with a control 
group(s), and randomised controlled trials [16]. The following factors were considered of importance during the quality assessment process: a clearly defined and specified study population, clearly defined and valid exposure and outcomes measures, adequate sample sizes, randomisation of participants, and a prespecified inclusion and exclusion criteria for being in the study. Two independent reviewers (WM and SF) conducted the quality assessment, and disagreements during the process were resolved through discussion by all the four authors. Answers to the questions of the two checklists gave an overall score of each article. Each paper was scored to a maximum of 12 points, with every 'yes' answer carrying 1 mark and 'no' answer carrying zero marks. An average of the scores from the two reviewers became the final quality score for each study. Quality was benchmarked as low if the average score of the two assessors was between 0 and 4 , moderate if the average score was from 5 to 8 , and high if average score was from 9 to 12 . However, no studies were excluded based on quality.

\section{Results}

Study selection and characteristics of the included studies Out of the initial 1309 (after 332 duplicates were removed), 13 studies (of over 2800 patients) met the inclusion criteria and were included to form the basis of the analysis. Most of the studies were excluded in the title and abstract screening stage because they were not relevant to the topic under review. Under full-text screening, studies were excluded because they were non-empirical, evaluating the feasibility and implementation of cervical cancer treatment methods, and assessing different stages of precancerous lesions and stages of cervical cancer in HIV-positive women. Most of the included studies (69.2\%) were published after the year 2010. The studies represented three regions, sub-Saharan Africa 8 (61.5\%), Asia 4 (30.8\%), and South America 1 (7.7\%), as indicated in Fig. 2.

The 13 included studies evaluated, assessed, or compared the effectiveness, treatment response, and outcomes of different cervical neoplasia and cervical cancer treatment methods for HIV-seropositive women. The results are presented in themes, that is, treatment methods of cervical neoplasia first, followed by treatment methods of confirmed cervical cancer. Five of the included studies (38.5\%) are prospective cohort, evaluating treatment response and toxicity to a combination of radiotherapy and chemotherapy, treatment with surgery and radiation, and treatment with loop electrosurgical excision procedure (LEEP). Four (30.8\%) retrospective cohort studies and one (7.7\%) evaluated the survival outcomes of chemotherapy, treatment outcomes of radiotherapy, and complications with LEEP, and compared clinical characteristics after radiation and chemotherapy. Two (15.4\%) randomised controlled trials compared the efficacy of LEEP vs cryotherapy and cryotherapy with no treatment. One $(7.7 \%)$ case study examined the results of a radical hysterectomy surgery on two different patients. All the 13 studies were almost consistent in defining their outcomes, such as treatment response, clinical/prognostic characteristics, survival response, and mortality rates. However, baseline characteristics of participants included in the studies were different, with age ranging from 18 years old to well above 55 years old. Sampling and recruitment of the participants were also different. In addition, participants had different stages of both precancerous lesions and cervical cancer, some were on highly active antiretroviral therapy (HAART), whilst others were not on HIV treatment, and the follow-up intervals were different as well (see Table 1). Research of cervical cancer treatment among HIV-seropositive
women by country

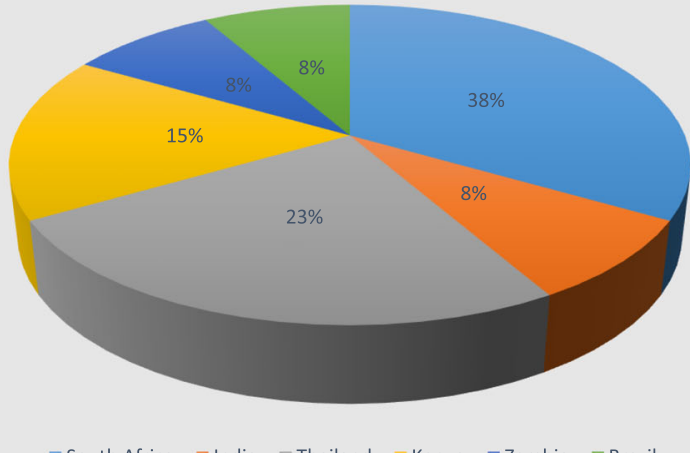

$\square$ South Africa $\square$ India $\square$ Thailand $\quad$ Kenya $\quad$ Zambia $=$ Brazil

Fig. 2 Research of cervical cancer treatment among HIV-seropositive women by country 


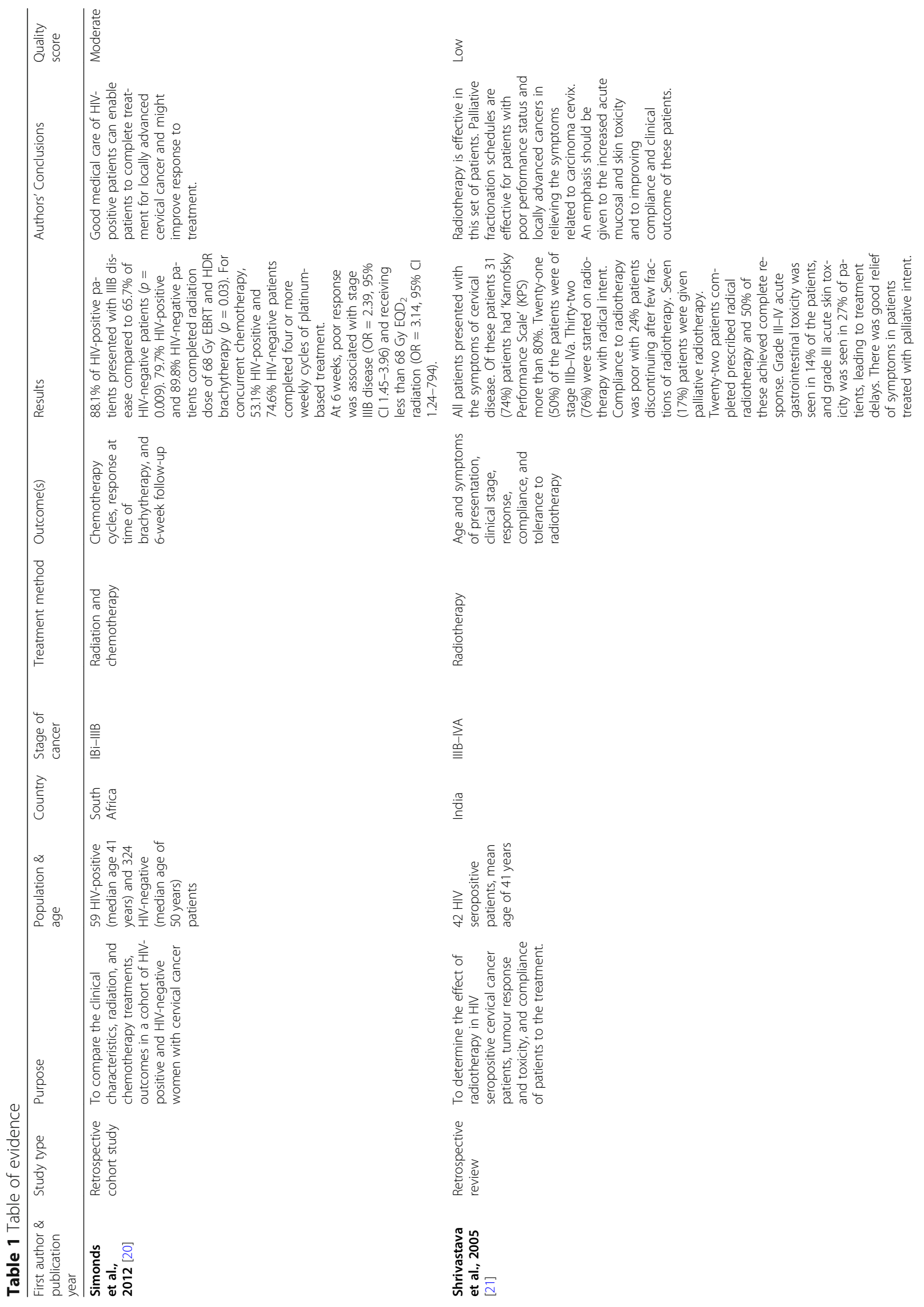




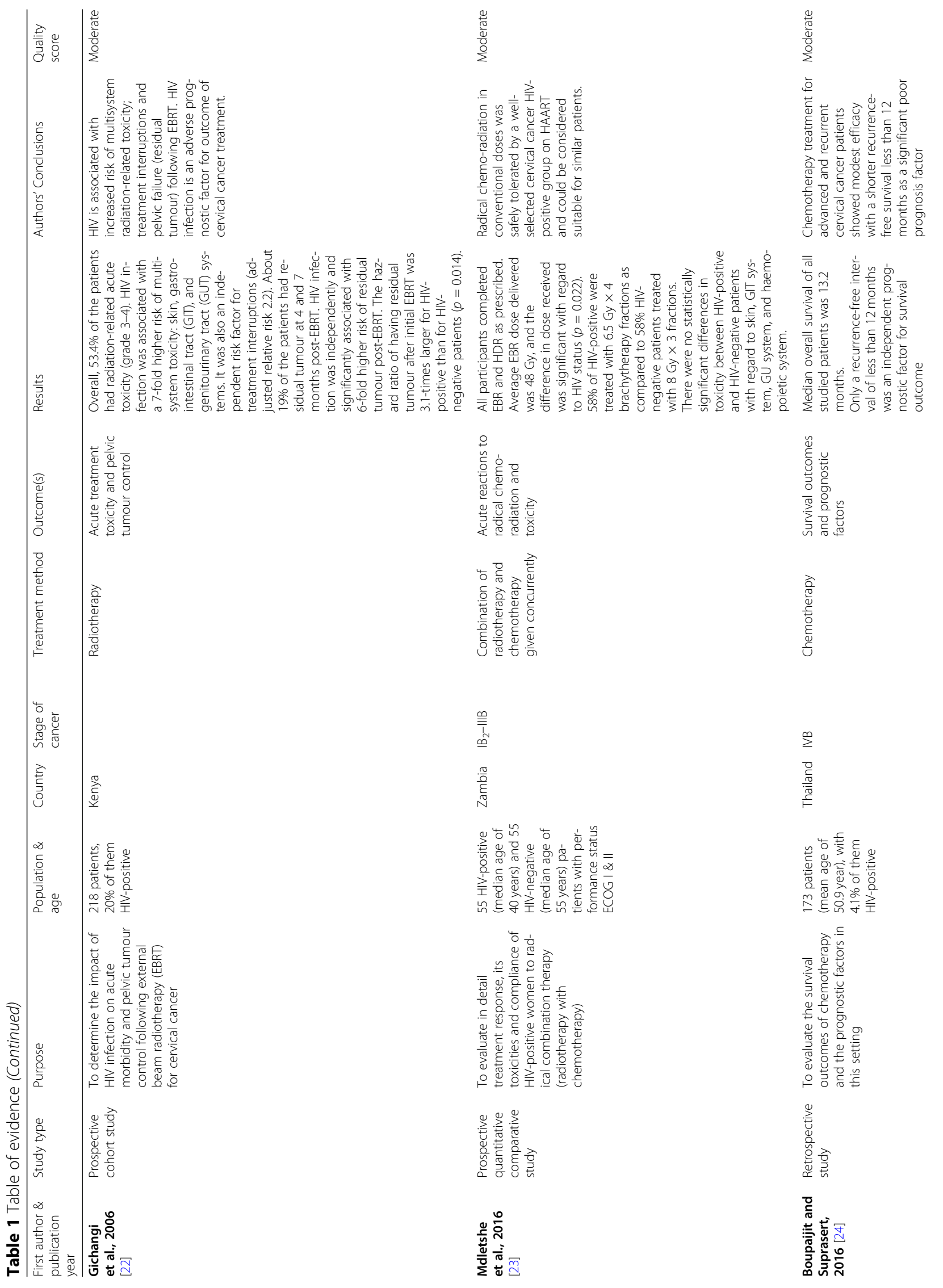




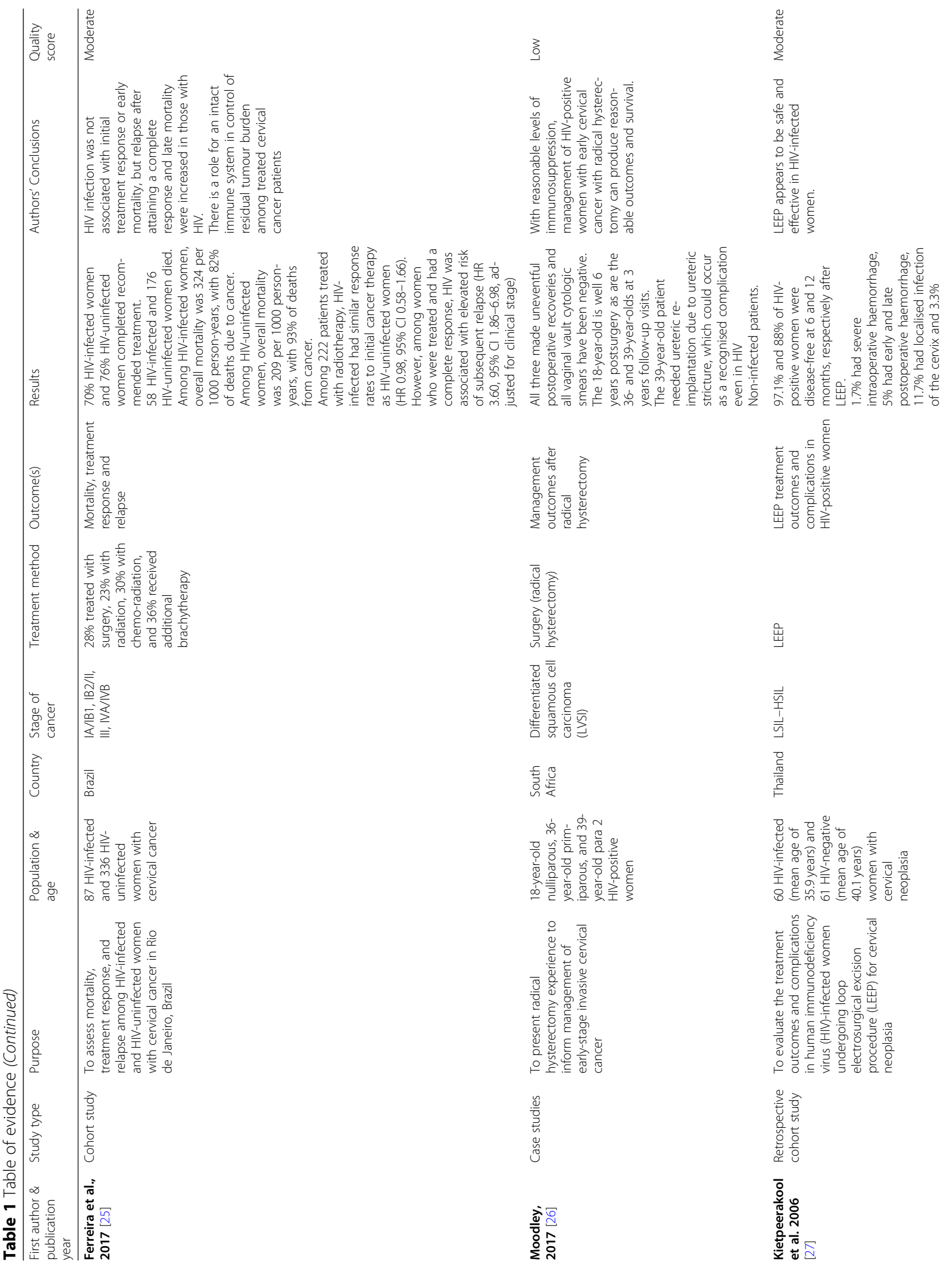




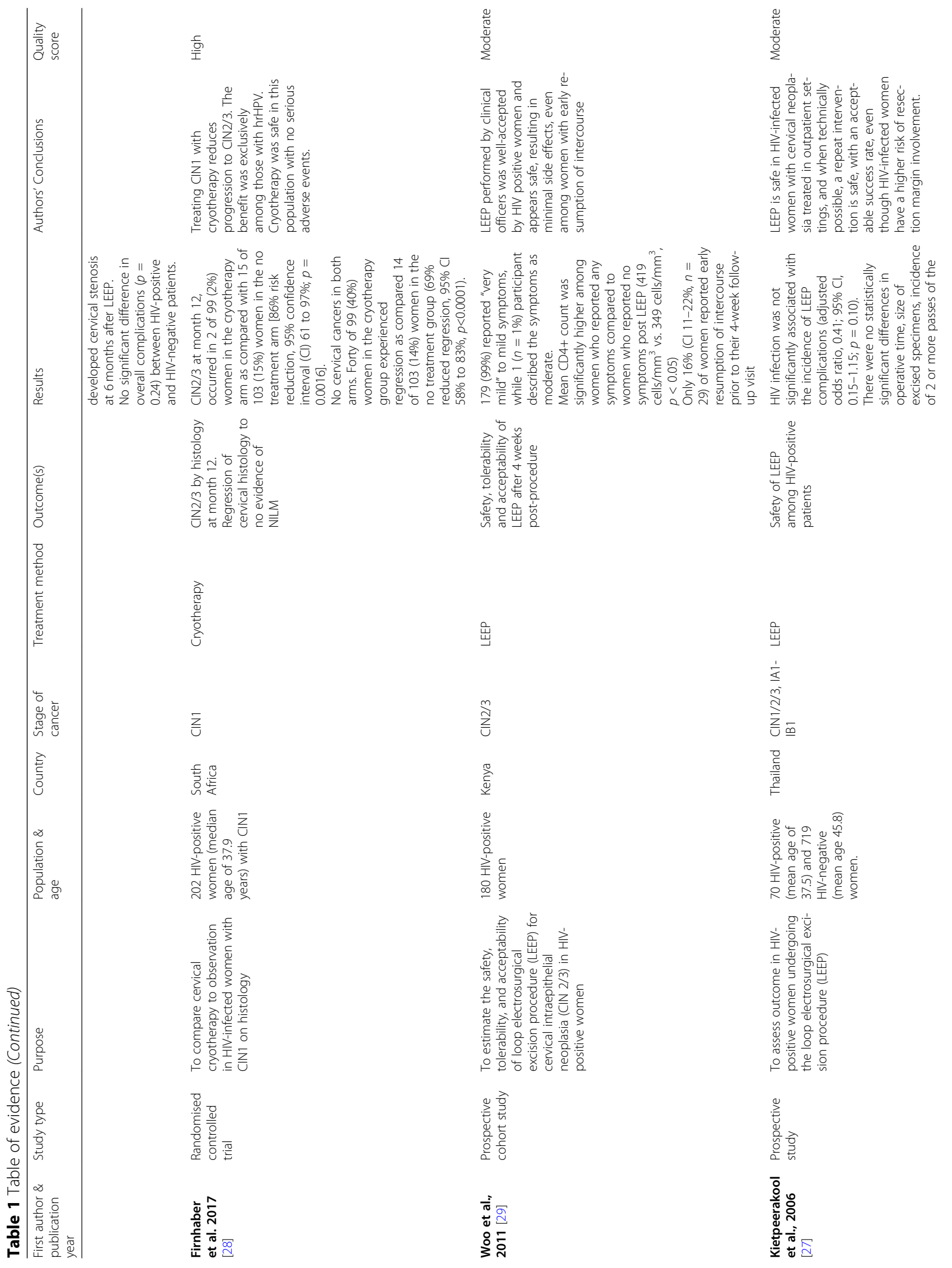




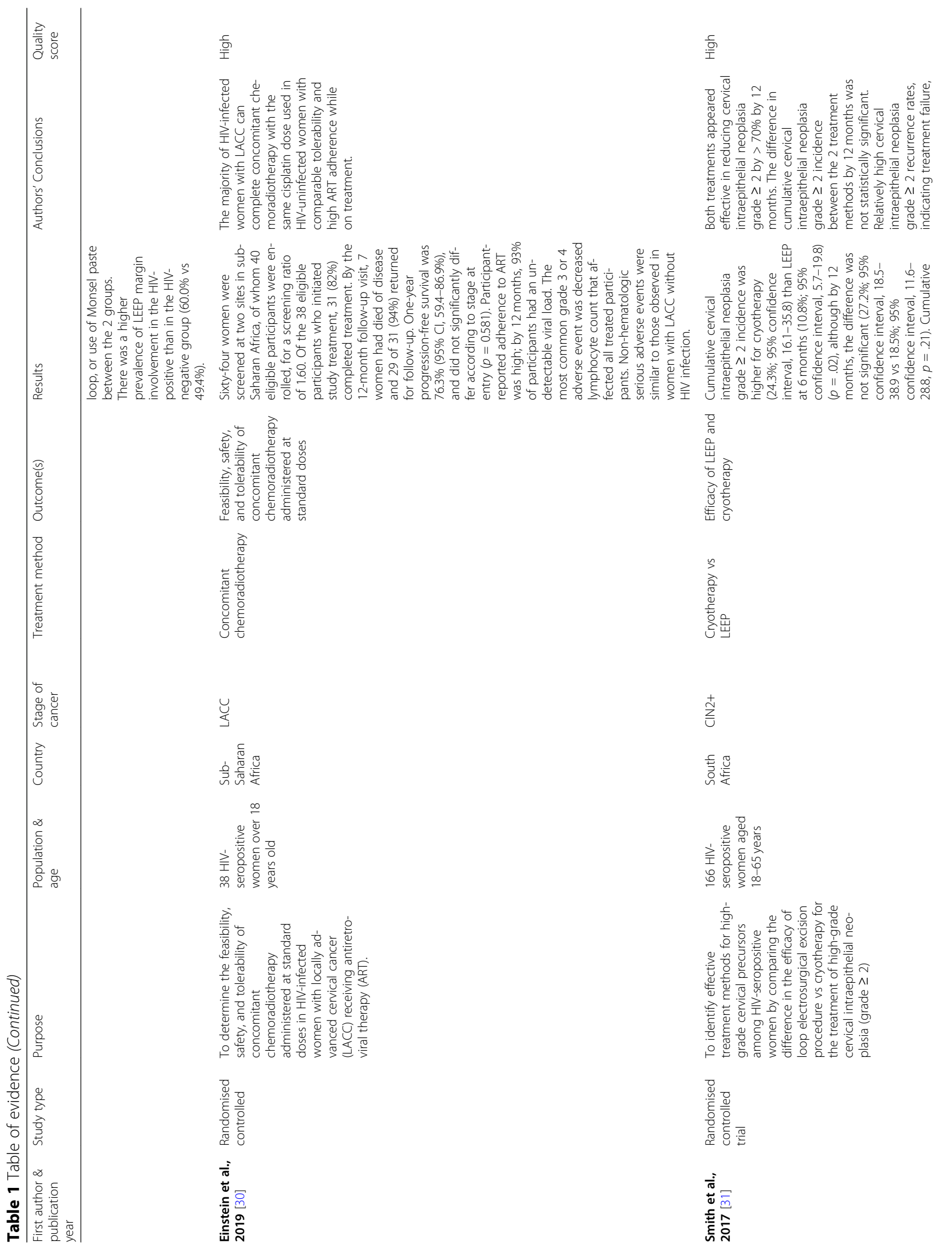




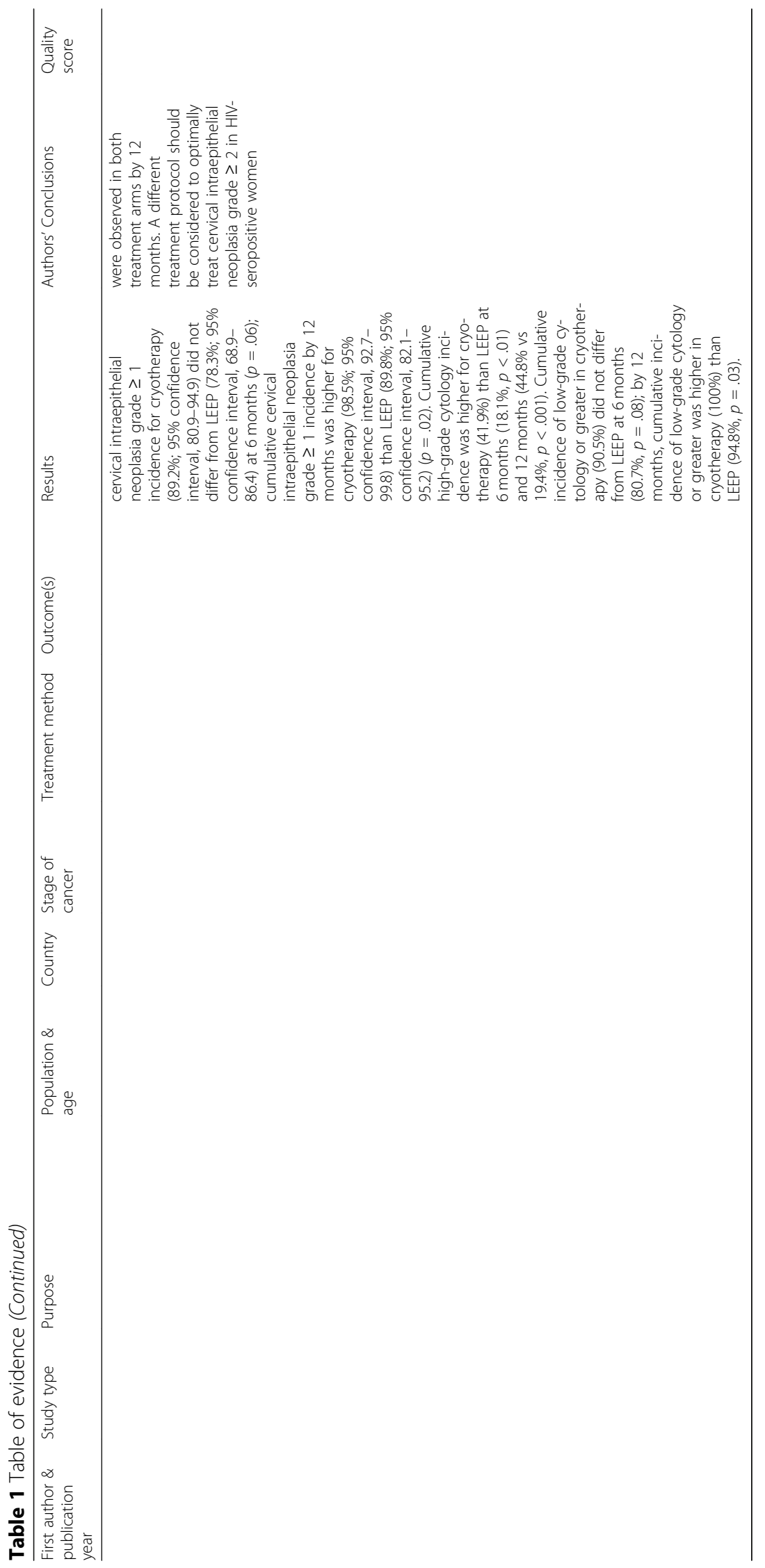




\section{Treatment options for cervical neoplasia for HIV seropositive women}

Five (38.5\%) of the 13 included studies evaluated efficacy, treatment outcomes, and complications in HIV-seropositive women with cervical neoplasia treated with LEEP or cryotherapy. Three studies evaluated LEEP [27, 29, 32], one compared cryotherapy with no treatment [28], and the other compared LEEP and cryotherapy to identify effective treatment [31].

\section{LEEP}

Three studies reviewing LEEP among HIV-positive women concluded that the procedure is safe and effective. A retrospective cohort study in Thailand evaluated treatment outcomes and complications of HIV-infected and HIV-negative women with a low-grade squamous intraepithelial lesion (LSIL) or high-grade squamous intraepithelial lesions (HSIL) undergoing LEEP [27]. The HIV-infected cohort had a mean age of 35.9 years as compared to 40.1 years of the HIV-negative cohort. After 6 and 12 months of LEEP, $97.1 \%$ and $88.0 \%$ of HIVinfected women had no cervical neoplasia, respectively. In terms of complications, there was no significant difference $(p=0.24)$ when compared to HIV-negative women [27]. These findings were almost similar to evidence generated in the same country 2 years later, which found out that there was no significant association between HIV and LEEP complications among women with cervical intraepithelial neoplasia (CIN) grades 1, 2, 3, and cervical cancer stage 1A1-1B1 [32]. In Kenya, a prospective cohort study also confirmed that LEEP was well tolerated and accepted by HIV-positive women who had CIN 2 and 3, with $99.0 \%$ of participants reporting 'very mild' symptoms of complications. Also, women with a higher mean $\mathrm{CD} 4+$ count were likely to report symptoms of complications as compared to women with lower mean CD4+ counts [29].

\section{Cryotherapy}

In a randomised controlled trial in South Africa among HIV-infected women with CIN1, treatment with cryotherapy was found to significantly reduce progression to CIN2/3. After 12 months, only $2 \%$ of women undergoing cryotherapy treatment as compared to $15 \%$ not receiving treatment (86\% risk reduction, 95\% CI 69-97\%, $p=$ $0.0016)$ progressed to CIN2/3. Regression was also significant in women receiving cryotherapy as compared to those not receiving treatment $69 \%$ reduced regression, 95\% CI 58-83\%, $p=0.0001)$ [28].

\section{Cryotherapy vs. LEEP}

To try and identify an effective treatment method between cryotherapy and LEEP for high-grade cervical precursors (CIN2+) among HIV-seropositive women, a randomised controlled trial was conducted in South Africa [31]. After 6 months of treatment, there was a higher cumulative CIN2+ incidence for cryotherapy (24.3\%, 95\% CI 16.1-35.8) as compared to LEEP (10.8\%, 95\% CI 5.7-19.8) at $p=0.02$. However, after 12 months of treatment, there was no significant difference between the two $(27.2 \%, 95 \%$ CI $18.5-38.9$ vs. $18.5 \%, 95 \% \mathrm{CI}$ 11.6-28.8) at $p=0.21$ [31]. Both cryotherapy and LEEP are effective in reducing $\mathrm{CIN} 2+$, and a choice might be based on available resources and expertise.

\section{Treatment options for cervical cancer for HIV seropositive women}

Treatment of cervical cancer with radiation, chemotherapy, concurrent treatment using radiotherapy and chemotherapy, and surgery among HIV-seropositive women was evaluated in $8(61.5 \%)$ out of the 13 included studies. The results of the treatment options are reported as themes as follows.

\section{Chemotherapy}

A retrospective study in Thailand on 173 HIV-positive and HIV-negative patients (with a mean age of 50.9 years) with stage IVB cervical cancer showed modest efficacy, with overall median survival among all patients of 13.2 months. The only independent prognostic survival outcome was a recurrence-free interval of fewer than 12 months [24]. In Brazil, HIV was found not to be associated with mortality due to cervical cancer during the first year post-treatment, but the association was significant after more than 1 to 2 years post-diagnosis (overall mortality: adj HR $=2.02$; 95\% CI 1.27-3.22; cancerspecific mortality $4.35,1.86-10.2)$ [25].

\section{Radiotherapy}

A retrospective review conducted in India to determine radiotherapy's effect on HIV-seropositive women of mean age of 41 years with cervical cancer stage IIIBIVA indicated that radiotherapy is effective, but compliance to the treatment is poor (with only $52.4 \%$ of women completing the prescribed radical radiotherapy and $50.0 \%$ of them achieving complete response) [21]. To overcome poor compliance, palliative radiotherapy schedules were prescribed, and these were identified to be effective for HIV-seropositive women with cervical cancer [21]. Despite it being effective, evidence has shown that those undergoing radiotherapy present with acute skin toxicity (grade III) and grade III-IV acute gastrointestinal toxicity [21]. These findings were supported by a prospective cohort study conducted in Kenya, which showed that there was a 7 -fold higher risk of developing multisystem (skin, gastrointestinal, and genitourinary) toxicity if HIV-infected and have undergone through radiotherapy [22]. This multisystem 
toxicity was found as a factor contributing to the interruption of treatment (adj. $R R=2.2$ ) [22]. Follow-ups at 4- and 7-months post-radiotherapy indicate that HIVseropositive is 6-fold at risk of having a residual tumour $(\mathrm{HR}=3.1, p=0.0014)$ as compared to patients who are HIV-negative [22]. This finding was in accord with what was suggested in Brazil where there was an elevated risk of subsequent relapse for HIV-seropositive women as compared to HIV-negative women ( $\mathrm{HR}=3.60 ; 95 \% \mathrm{CI}$ 1.86-6.98) [25].

\section{Radiation and chemotherapy}

To compare the clinical characteristic outcomes after radiation and chemotherapy among HIV-positive (median age of 41 years) and HIV-negative women (median age of 50 years) with cancer stage IBi-IIIB, a retrospective cohort study was conducted in South Africa [20]. Treatment completion rates between the two patient cohorts were different, with $79.7 \%$ of HIV-positive and $89.8 \%$ HIV-negative completing their radiation dose and brachytherapy $(p=0.03)$. For concurrent chemotherapy, only 53.1\% HIV-positive and 74.6\% HIV-negative managed to complete 4 or more weekly cycles. After 6 weeks, poor response to treatment was significantly associated with stage IIIB (OR $=2.39$, 95\% CI 1.45-3.96) and receiving less than recommended radiation dose $(\mathrm{OR}=$ 3.14, 95\% CI 1.24-7.94) [20].

\section{Combination of radiotherapy and chemotherapy}

A prospective quantitative comparative study in Zambia evaluated the treatment response, treatment toxicities, and compliance to radical chemo-radiation among both HIV-positive (median age of 40 years) and HIV-negative (median age of 55 years) women with stage $\mathrm{IB}_{2}$-IIIB cancer [23]. As opposed to a failure to complete treatment as indicated by evidence in South Africa [20], all participants in this prospective study completed their treatments. Well-selected HIV-positive cervical cancer patients on HAART can safely tolerate radical chemoradiation in conventional doses [23]. The difference in chemo-radiation doses $(6.5$ Gy $\times 4$ for $58 \%$ of HIVpositive women vs. $8 \mathrm{~Gy} \times 3$ for $58 \%$ of HIV-negative women) was significant to HIV status $(p=0.022)$. In terms of toxicity (regarding GIT system, skin, haemopoietic system, and GU system), there were no significant differences between HIV-positive and HIV-negative patients [23]. In a study of 38 HIV-positive women with locally advanced cervical cancer, the safety, tolerability, and feasibility of concomitant chemoradiotherapy were assessed in two sites in sub-Saharan Africa. Results indicated that HIV-infected women (82\%) who adhere to ART can tolerate and complete concomitant chemoradiotherapy as HIV-negative women. After 1 year with 7 women dead due to cervical cancer, 29 of the remaining
31 (94\%) returned for a scheduled clinical visit and progression-free survival was at $76.3 \%$ (95\% CI, 59.486.9\%) [30].

\section{Surgery (radical hysterectomy)}

Three case studies in South Africa of HIV-positive women with LVSI, an 18-year-old nulliparous, 36-yearold primiparous, and 39-year-old para-2, examined the radical hysterectomy to inform management of earlystage invasive cancer [26]. After 6 years post-surgery, the 18 -year old has recovered, and all the vaginal vault cytologic smears have come negative. At 3 years of follow-up visits, both the 36- and 39-year olds have also recovered and with negative vaginal vault cytologic smears [26].

\section{Quality assessment of included studies}

Few studies $(n=2,15.4 \%)$ were determined to be of 'high' quality using a combination of the modified Newcastle-Ottawa Quality Assessment Scale and the NIH Study Quality Assessment Tools for observational cohort cross-sectional case-control and before-after studies $[18,19]$. Most of the studies $(n=9,69.2 \%)$ were of 'moderate' quality, and two (15.4\%) were of 'low' quality. Adequate randomisation, enough sample sizes, prespecified inclusion and exclusion criteria, specified study population, and clearly defined exposure and outcomes measures were all available in both controlled interventions [28, 31], and this increased the confidence that the reported results might have been attributable to the intervention than the difference in groups. For the before-after studies, 6 out of 9 studies had a control group [20, 23, 25, 27, 29, 32], specified inclusion and exclusion criteria, and defined exposure and outcome measures, and this also increased confidence that the reported improvements between before and after evaluations were not merely by chance. However, different participants' selection, small sample sizes, and short follow-up periods among other studies [20-22, 28, 31], including two descriptive studies $[24,26]$ that did not mention how study participants were chosen or how exposure and outcomes measures were defined, might require their results to be interpreted with caution. Also, inadequate follow-up periods, failure to measure or include confounders in analyses, and lack of validity of reported outcomes might have resulted in some studies overestimated the effectiveness of the reported interventions.

\section{Discussion}

This systematic review aimed to synthesise available evidence on treatment modalities for both cervical neoplasia and cervical cancer in HIV-seropositive women in developing countries. Most cervical cancer patients are reported to be diagnosed at an advanced stage of the 
disease because of the lack of coordinated and systematic screening $[9,10]$. Besides, lack of optimal treatment regimen due to factors such as lack of infrastructure, financial, and human resources has been found to contribute to poor outcomes of treatment among HIVseropositive women in developing countries [10, 13]. The findings of this systematic review have shown that the available cervical cancer treatments, radiotherapy, chemotherapy, chemoradiation, and surgery appear to be effective for HIV-seropositive patients and are the same treatments being used for HIV-negative patients as well as in developed countries. This review has also shown that opportunities to improve cervical neoplasia and cervical cancer management in HIV-positive women exist. However, developing countries need to prioritise early diagnosis and treatment of precancerous lesions to reduce cervical cancer and align with 2030 Sustainable Development Goals to reduce non-communicable diseases mortality. As most developing countries put plans and measures in place for universal health coverage by 2030 , it is paramount that benefits packages to be offered should include cervical cancer screening, HPV vaccination, testing, and treatment especially for HIV-positive women if they are to achieve the same impact as developed countries.

The introduction of life-long antiretroviral (ART) has been found to moderately reduce HPV infection incidences [33]. Despite the moderate effect on HPV infection, ART is prolonging the life span of those infected with HIV, thereby granting time for the development of cervical neoplasia and cervical cancer especially in countries with not well-established cervical cancer screening programs. This systematic review has confirmed that the available treatments for both cervical neoplasia and cervical cancer (if detected early) among HIV-seropositive women appear to be effective. However, clinical, methodological, and statistical heterogeneity, such as participants' baseline characteristics, immunosuppressive status, follow-up time, randomisation versus non-randomisation, sample sizes, and statistical calculations, among the 10 studies, might explain some the differences in the findings. In this review, almost all the included studies had HIVseropositive women who were younger than HIV-negative women were.

This systematic review demonstrated that LEEP and cryotherapy treatments have the possibility of reducing progression from LSIL to HSIL as well as causing regression of cervical neoplasia [27, 29, 31, 32]. However, this treatment benefit was exclusively significant among women with high-risk HPV and might point to a need for further multicentre research to explore the reasons for such a finding.

In as much as LEEP was reported to be safe, several complications, such as severe intraoperative haemorrhage, early and late postoperative haemorrhage, localised infection of the cervix, and cervical stenosis, were experienced in both HIV-positive and HIV-negative women although the difference was insignificant [27]. Despite no difference in complications between HIV-positive and HIV-negative women, further research on reasons for such complications need to be assessed and explored to inform best clinical practices.

In India, treatment with radiotherapy was seen to be effective among HIV-seropositive women with cervical cancer stage IIIB-IVA [21], and these findings were supported by evidence from Kenya [22]. However, the associated acute treatment toxicity of radiotherapy among HIV-positive women was seen to be an independent significant risk factor that interrupts or delay treatment resulting in most of these women not completing their prescribed treatments [22]. Acute gastrointestinal, skin, and genitourinary tract toxicity is the most prominent radiation-related acute toxicities and is associated with HIV [21, 22]. These multisystem acute toxicity findings contrast with what was identified in a radical chemoradiation prospective study which reported no statistically significant differences between HIV-positive and HIVnegative patients [23]. Therefore, further studies examining patients' baseline characteristics such as time of HAART or CD4+ counts will need to be conducted to analyse why studies are reporting different findings.

Being HIV-seropositive prevents the success of radiotherapy as most patients will not complete prescribed treatment due to associated multisystem toxicities hence resulting in poor response and outcomes in some cases. After 7 months post-radiotherapy, HIV-seropositive women were 3.1 times likely to have a residual tumour as compared to HIV-negative [22]. These findings indicate that completing radiation is a predictor of treatment response among HIV-seropositive women [20,34]. Palliative radiotherapy fractionation has been reported to be effective in HIV-seropositive patients with poor performance and advanced cancer [22], but having an intact immune system and a higher CD4+ count is a positive indicator to treatment response and reduction of tumour [25].

Despite completing prescribed treatment being an indicator of treatment response in radiotherapy [21, 22], evidence on chemotherapy indicates that treatment completion did not have a greater effect or impact on the response after 6 weeks as compared to radiotherapy [20]. Besides, cervical cancer stage IIIB was indicated to be associated with poor chemo-radiation after 6 weeks [20], and this might suggest that offering a full dose of radiation coupled with good medical care in terms of associated toxicities [21, 22] might be beneficial to HIV-seropositive with advanced cervical cancer. This suggestion is supported by findings that show that chemo-radiation incremental benefit as compared to radiotherapy is minimal [35]. However, these findings required further studies to 
be conducted with large numbers of patients to assess the reported treatment outcomes because the evidence in Zambia has indicated that conventional doses of radical chemo-radiation are well tolerated and effective for HIVseropositive women who are on HAART [23].

Three radical hysterectomies on reasonably stable immunosuppressive HIV-seropositive patients with cervical cancer stage IB-IIA appeared to produce better treatment and survival outcomes, with all three patients having negative vault cytologic smears after 3- and 6-years post-surgery [26]. However, because of the few patients reported in this radical hysterectomy study, there might be a need to explore further the impact of this treatment and associated outcomes.

\section{Limitations}

Despite the overall quality of the included studies being moderate, some of the reported results were affected by the risk of bias associated with the comparability of effects, populations, and information including lack of explanations on the conducted statistical analyses. By limiting our study searches to those reported in English, this systematic review might have missed some relevant studies published in other languages.

\section{Conclusions}

Those infected with HIV were younger and have advanced disease as compared to those who were HIVnegative [20-25, 27-29, 31, 32]. In as much as the mass $\mathrm{HPV}$ vaccination is targeting 9- to 13-year-old young girls, it can be argued, based on the findings from this review, that developing countries must offer targeted vaccination [36] to HIV-positive adolescents and young women between the ages of 13 and 26 years through already established HIV clinics, to increase vaccination coverage and consolidated potential benefits. This is in line with the Guidelines for Prevention and Treatment of Opportunistic Infections in HIV-Infected Adults and Adolescents [37]. Offering routine cervical cancer screening and HPV testing in HIV clinics might help in early identification of these at high-risk women who are relatively ignorant and lack knowledge about cervical cancer risk factors. Facilitation and putting HIV-infected people on life-long ART is of importance and has been found to have a positive impact on cervical cancer treatment response.

\section{Implications of the review's results to evidence- based health care}

Based on this review, the following key messages on the reliability of the findings have emerged:

- Both cervical neoplasia and cervical cancer in HIVseropositive women are treatable with the available treatment, and better outcomes are associated with early diagnosis and treatment availability. Also, these are the same treatments that are available in developed countries and have made a tremendous impact. However, the differences between developing and developed countries are around lack of optimal treatment regimen and underutilisation of available cervical cancer services due to cost, lack of knowledge, lack of infrastructure, and human resources that continue to hamper developing countries. There is a need for good clinical management of HIVseropositive women undergoing chemo-radiation to manage multisystem toxicities that have a bearing on treatment completion, prognostic, and survival outcomes. Research on cervical cancer management of HIV-seropositive patients focusing on the quality of life of those treated, the effectiveness of the treatment method considering CD4+ count, and ART is required.

- As HPV infections continue to be high among HIVpositive women regardless of ART, primary prevention through HPV vaccination is critically needed among young HIV-positive girls at the recommended ages since the vaccine is safe and beneficial to them. Most HIV-seropositive women with cervical cancer are young, and screening from the age of 15 years, taking into consideration early sexual debut and high HIV incidence, might increase early identification of at-risk young women. There is a need to strengthen health systems by establishing robust and regular cervical cancer screening and HPV testing beginning at age 21 in HIV testing and treatment clinics. Multicentre research on early screening of young women is required to inform feasibility, appropriateness, meaningfulness, and cost-effectiveness.

\section{Supplementary information}

Supplementary information accompanies this paper at https://doi.org/10. 1186/s13643-020-01345-2.

Additional file 1. PubMed and OvidSP (MEDLINE and Embase) Search Strategies. This file contains two examples of the search strategies used to search for studies that were included in this literature review. The search strategies are for PubMed, MEDLINE and Embase databases.

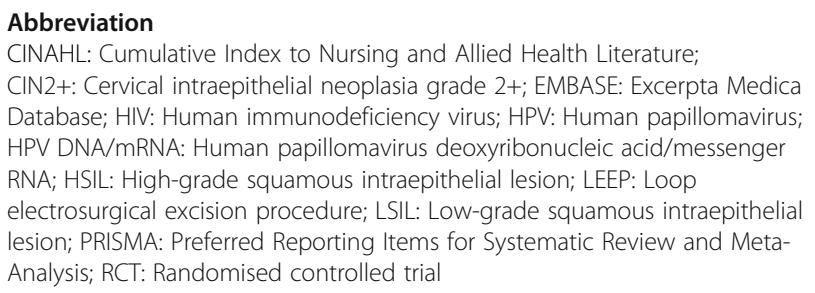




\section{Acknowledgements}

The review team is grateful to Rutendo Chinomona-Mapanga for her comments and insights on the systematic review.

\section{Authors' contributions}

The principal reviewer WM designed this systematic review including its registration with PROSPERO. As the principal reviewer, WM led the literature searches, screening process, data extraction, and analysis. All the team members participated in the literature search and screening, data extraction, and analysis. The final version of the manuscript was critically appraised and approved by all the authors.

\section{Authors' information}

WM is a Public Health Specialist and Cancer Clinical Epidemiologist, and this study was part of his Ph.D. at the School of Health Systems and Public Health, University of Pretoria. WM's research was focusing on cervical cancer and HIV in developing countries. ES is the Head of the South African National Cancer Registry. BGB is a professor of Biostatistics at the Department of Epidemiology and Biostatistics at the University of Pretoria. SF is a professor of Biostatistics and Epidemiology at the Faculty of Health Sciences, University of Fort Hare.

\section{Funding}

The research project, which this systematic review is part of, has been funded by the University of Pretoria (program code 10260404). The University of Pretoria had no role in designing this systematic review, how it was conducted, writing of the manuscript, or the decision to publish this systematic review.

\section{Availability of data and materials}

The analysed data and materials are included in this publication.

\section{Ethics approval and consent to participate}

This systematic review is part of a bigger research project. Ethical approval for the project was obtained from the Research Ethics Committee of the Faculty of Health Sciences, University of Pretoria (ethics reference number: 146/2016)

\section{Consent for publication}

Not applicable.

\section{Competing interests}

The authors declare that they have no competing interests.

\section{Author details}

${ }^{1}$ School of Health Systems and Public Health, Epidemiology \& Biostatistics, University of Pretoria, 5-10 H.W. Snyman Building, Pretoria, South Africa. ${ }^{2}$ Non-Communicable Diseases Research (NCDR) Division of the Wits Health Consortium, Faculty of Health Sciences, University of the Witwatersrand, Johannesburg, South Africa. ${ }^{3}$ Brooklyn, South Africa. ${ }^{4}$ Cancer Epidemiology Research Group, National Cancer Registry, National Health Laboratory Service, Johannesburg, South Africa. ${ }^{5}$ Community Medicine Unit, School of Public Health, University of the Witwatersrand, Johannesburg, South Africa. ${ }^{6}$ Faculty of Health Sciences, The University of Fort Hare, P.O. Box 1054, 45 Church Street, Gasson Building, 7th Floor, East London 5201, South Africa.

\section{Received: 29 August 2019 Accepted: 30 March 2020}

\section{Published online: 10 April 2020}

\section{References}

1. Shields TS, Brinton LA, Burk RD, et al. A case-control study of risk factors for invasive cervical cancer among U.S women exposed to oncogenic types of human papillomavirus. Cancer Epidemiology Biomakers Prevention. 2004;13: 1574-82.

2. Burd EM. Human papillomavirus and cervical cancer. Clin Microbiol Rev. 2003;16(1):1-17. https://doi.org/10.1128/CMR.16.1.1-17.2003.

3. Word Health Organisation., Institut Catala' d'Oncologia (ICO). Human Papillomavirus and related cancers, HPV information centre. Gevena: WHO/ ICO; 2010.

4. Bruni L, Diaz M, Castellsagué X, Ferrer E, Bosch FX, de Sanjosé S. Cervical human papillomavirus prevalence in 5 continents: meta-analysis of 1 million women with normal cytological findings. J Infect Dis. 2010 Dec 15;202(12): 1789-99.

5. Chirenje ZM. HIV and cancer of the cervix. Best Pract Res Clin Obstet Gynaecol. 2005;19:269-76.

6. Franceschi $\mathrm{S}$, Jaffe $\mathrm{H}$. Cervical cancer screening of women living with HIV infection: a must in the era of antiretroviral therapy. Clin Infect Dis. 2007; 45(4):510-3. https://doi.org/10.1086/520022.

7. Ghebre RG, Grover S, Xu MJ, Chuang LT, Simonds H. Cervical cancer control in HIV-infected women: past, present and future. Gynecol Oncol Rep. 2017; 21:101-8.

8. Shrestha AD, Neupane D, Vedsted P, Kallestrup P. Cervical cancer prevalence, incidence and mortality in low and middle income countries: a systematic review. Asian Pac J Cancer Prev. 2018;19(2):319-24.

9. Santesso N, Mustafa RA, Schünemann HJ, Arbyn M, Blumenthal PD, Cain J, Chirenje $M$, et al. World Health Organization Guidelines for treatment of cervical intraepithelial neoplasia 2-3 and screen-and-treat strategies to prevent cervical cancer. Int J Gynaecol Obstet. 2016;132(2):252-8.

10. Coleman JS, Cespedes MS, Cu-Uvin S, Kosgei RJ, Maloba M, Anderson J, et al. An insight into cervical cancer screening and treatment capacity in sub Saharan Africa. J Low Genit Tract Dis. 2016;20(1):31-7.

11. Elit $L$, Fyles AW, Devries MC, et al. Follow-up for women after treatment for cervical cancer: a systematic review. Gynecol Oncol. 2009;114:528.

12. National Comprehensive Cancer Network (NCCN). NCCN Clinical practice guidelines in oncology. http://www.nccn.org/professionals/physician_gls/f_ guidelines.asp.

13. Finocchario-Kessler S, Wexler C, Maloba M, Mabachi N, Ndikum-Moffor F, Bukusi E. Cervical cancer prevention and treatment research in Africa: a systematic review from a public health perspective. BMC Womens Health. 2016;16:29.

14. Sherris J, Herdman C, Elias C. Cervical cancer in the developing world. West J Med. 2001;175(4):231-3.

15. Fletcher FE, Vidrine DJ, Tami-Maury I, Danysh HE, King RM, Buchberg M, Arduino RC, Gritz ER. Cervical cancer screening adherence among HIVpositive female smokers from a comprehensive HIV clinic. AIDS Behav. 2014; 18(3):544-54.

16. Mapanga W, Elhakeem A, Feresu SA, Maseko F, Chipato T. Prevention of cervical cancer in HIV-seropositive women from developing countries: a systematic review protocol. Syst Rev. 2017;6(1):91. https://doi.org/10.1186/ s13643-017-0484-9.

17. Moher D, Liberati A, Tetzlaff J, Altman DG, The PRISMA Group. Preferred Reporting Items for Systematic Reviews and Meta-Analyses: the PRISMA statement. PLoS Med. 2009;6(7):e1000097. https://doi.org/10.1371/journal. pmed.1000097.

18. NIH Study Quality Assessment Tools. Available at https://www.nhlbi.nih.gov/ health-topics/study-quality-assessment-tools [Accessed 2 October 2018].

19. Wells GA, Shea B, O'Connell D, Peterson J, Welch V, Losos M, Tugwell P. The Newcastle-Ottawa Quality Assessment Scale. Available at http://www.ohri. ca/programs/clinical_epidemiology/oxford.asp [Accessed 11 Oct 2016].

20. Simonds HM, Wright JD, du Toit N, Neugut Al, Jacobson JS. Completion of and early response to chemoradiation among HIV-positive and HIV-negative patients with locally advanced cervical carcinoma in South Africa. Cancer. 2012;118(11):2971-9.

21. Shrivastava SK, Engineer R, Rajadhyaksha S, Dinshaw KA. HIV infection and invasive cervical cancers, treatment with radiation therapy: toxicity and outcome. Radiother Oncol. 2005;74(1):31-5.

22. Gichangi P, Bwayo J, Estambale B, Rogo K, Njuguna E, Ojwang S, Temmerman M. HIV impact on acute morbidity and pelvic tumor control following radiotherapy for cervical cancer. Gynecol Oncol. 2006;100(2):405-11.

23. Mdletshea S, Munkupab H, Lishimpib K. Acute toxicity in cervical cancer HIV-positive vs. HIV-negative patients treated by radical chemo-radiation in Zambia. South Afr J Gynaecol Oncol. 2016;8(2):37-41.

24. Boupaijit K, Suprasert P. Survival outcomes of advanced and recurrent cervical cancer patients treated with chemotherapy: experience of Northern Tertiary Care Hospital in Thailand. Asian Pac J Cancer Prev. 2016;17(3):1123-7.

25. Ferreira MP, Coghill AE, Chaves CB, Bergmann A, Thuler LC, Soares EA, Pfeiffer RM, Engels EZ, Soares MA. Outcomes of cervical cancer among HIVinfected and uninfected women treated at the Brazilian National Institute of Cancer (2001-2013). AIDS. 2017;31(4):523-31.

26. Moodley M. Radical hysterectomy for cervical cancer amongst women infected with the human immunodeficiency virus. Int J Gynecol Cancer. 2007;17:1264-5 
27. Kietpeerakool C, Srisomboon J, Suprasert P, Phongnarisorn C, Charoenkwan K, Cheewakriangkrai C, Siriaree S, Tantipalakorn C, Pantusart A. Outcomes of loop electrosurgical excision procedure for cervical neoplasia in human immunodeficiency virus-infected women. Int J Gynecol Cancer. 2006;16:1082-8. https://doi.org/10.1111/j.1525-1438.2006.00518.x.

28. Firnhaber C, Swarts A, Goeieman B, Rakhombe N, Mulongo M, Williamson AL, Michelow P, Ramotshela S, Faesen M, Levin S, Wilkin T. Cryotherapy reduces progression of cervical intraepithelial neoplasia grade 1 in South African HIV-infected women: a randomized, controlled trial. J Acquir Immune Defic Syndr. 2017;76:532-8.

29. Woo VG, Cohen CR, Bukusi EA, Huchko M. Loop electrosurgical excision procedure: safety and tolerability among human immunodeficiency viruspositive Kenyan women. Obstet Gynecol. 2011;118(3):554-9.

30. Einstein MH, Ndlovu N, Lee J, Stier EA, Kotzen J, Garg M, Whitney K, Krown SE. Cisplatin and radiation therapy in HIV-positive women with locally advanced cervical cancer in sub-Saharan Africa: a phase II study of the AIDS malignancy consortium. Gynecologic Oncology. 2019;153(1):20-5.

31. Smith JS, Sanusi B, Swarts A, Faesen M, Levin S, Goeieman B, Ramotshela S, Rakhombe N, Williamson AL, Michelow P, Omar T, Hudgens MG, Firnhaber C. A randomized clinical trial comparing cervical dysplasia treatment with cryotherapy vs loop electrosurgical excision procedure in HIV-seropositive women from Johannesburg, South Africa. Am J Obstet Gynecol. 2017;217: 183.e1-11.

32. Kietpeerakool C, Suprasert P, Srisomboon J. Outcomes of loop electrosurgical excision procedure for HIV-positive women in a low-resource outpatient setting. Int J Gynecol Obstet. 2009:105:10-3.

33. Bower M, Mazhar D, Stebbing J. Should cervical cancer be an acquired immunodeficiency syndrome-defining cancer? J Clin Oncol. 2006;24(16): 2417-9

34. Oehler-Janne C, Huguet F, Provencher S, et al. HIV-specific differences in outcome of squamous cell carcinoma of the anal canal: a multicentric cohort study of HIV-positive patients receiving highly active antiretroviral therapy. J Clin Oncol. 2008;26(15):2550-7.

35. Chemoradiotherapy for Cervical Cancer MetaAnalysis Collaboration (CCCMAC). Reducing uncertainties about the effects of chemoradiotherapy for cervical cancer: Individual patient data metaanalysis. Cochrane Database Syst Rev. 2010;1:CD008285.

36. Tan N, Sharma M, Winer R, Galloway D, Rees H, Barnabas RV. Modelestimated effectiveness of single dose 9-valent HPV vaccination for HIVpositive and HIV-negative females in South Africa. Vaccine. 2018;36:4830-6.

37. Masur H, Brooks JT, Benson CA, Holmes KK, Pau AK, Kaplan JE. Prevention and treatment of opportunistic infections in HIV-infected adults and adolescents: updated guidelines from the centers for disease control and prevention, National Institutes of Health, and HIV Medicine Association of the Infectious Diseases Society of America. Clin Infect Dis. 2014;58(9):1308-11.

\section{Publisher's Note}

Springer Nature remains neutral with regard to jurisdictional claims in published maps and institutional affiliations.

Ready to submit your research? Choose BMC and benefit from:

- fast, convenient online submission

- thorough peer review by experienced researchers in your field

- rapid publication on acceptance

- support for research data, including large and complex data types

- gold Open Access which fosters wider collaboration and increased citations

- maximum visibility for your research: over $100 \mathrm{M}$ website views per year

At $\mathrm{BMC}$, research is always in progress.

Learn more biomedcentral.com/submissions 